\title{
Plasma convection in the magnetotail lobes: statistical results from Cluster EDI measurements
}

\author{
S. Haaland ${ }^{1,2, *}$, G. Paschmann ${ }^{2}$, M. Förster ${ }^{3}$, J. Quinn ${ }^{4, *}$, R. Torbert ${ }^{4}$, H. Vaith ${ }^{4}$, P. Puhl-Quinn ${ }^{4}$, and C. Kletzing ${ }^{5}$ \\ ${ }^{1}$ Max-Planck-Institut für extraterrestrische Physik, Garching, Germany \\ ${ }^{2}$ Department of Physics, University of Bergen, Norway \\ ${ }^{3}$ GeoForschungsZentrum Potsdam, Potsdam, Germany \\ ${ }^{4}$ University of New Hampshire, Durham, NH 03824, USA \\ ${ }^{5}$ University of Iowa, Iowa City, IA 52242, USA \\ *now at: Max-Planck-Institut für Sonnensystemforschung, Lindau, Germany \\ *** now at: Boston University, Boston, MA 02215, USA
}

Received: 4 April 2008 - Revised: 26 May 2008 - Accepted: 31 May 2008 - Published: 5 August 2008

\begin{abstract}
A major part of the plasma in the Earth's magnetotail is populated through transport of plasma from the solar wind via the magnetotail lobes. In this paper, we present a statistical study of plasma convection in the lobes for different directions of the interplanetary magnetic field and for different geomagnetic disturbance levels. The data set used in this study consists of roughly 340000 one-minute vector measurements of the plasma convection from the Cluster Electron Drift Instrument (EDI) obtained during the period February 2001 to June 2007. The results show that both convection magnitude and direction are largely controlled by the interplanetary magnetic field (IMF). For a southward IMF, there is a strong convection towards the central plasma sheet with convection velocities around $10 \mathrm{~km} \mathrm{~s}^{-1}$. During periods of northward IMF, the lobe convection is almost stagnant. A $B_{y}$ dominated IMF causes a rotation of the convection patterns in the tail with an oppositely directed dawn-dusk component of the convection for the northern and southern lobe. Our results also show that there is an overall persistent duskward component, which is most likely a result of conductivity gradients in the footpoints of the magnetic field lines in the ionosphere.
\end{abstract}

Keywords. Magnetospheric physics (Magnetospheric configuration and dynamics; Plasma convection; Solar windmagnetosphere interactions)

Correspondence to: S. Haaland

(stein.haaland@ift.uib.no)

\section{Introduction}

The Earth's magnetosphere is a dynamical system primarily controlled by the solar wind and the interplanetary magnetic field (IMF). When the IMF has a southward component, it can reconnect with the Earth's geomagnetic field on the dayside magnetopause. The recently opened magnetic field lines are dragged by the solar wind across the polar caps into the magnetotail lobes and then eventually into the central plasma sheet of the magnetotail where the field lines eventually reconnect and return towards the Earth (Dungey, 1961). The convection of plasma through the magnetotail lobes can therefore be used as a proxy for the energy transfer from the solar wind into the magnetosphere.

The location of the dayside reconnection line and the region of plasma entry into the magnetotail are also modulated by the IMF $B_{y}$ component. In the Northern Hemisphere a positive (negative) $B_{y}$ will cause a displacement of the reconnection region so that the newly opened flux tubes are transported towards dawn (dusk), and oppositely for the Southern Hemisphere (e.g. Cowley et al., 1991). This $B_{y}$ influence is also reflected in the convection in the lobes (Gosling et al., 1984, 1985; Noda et al., 2003), the ecliptic plane (Baumjohann and Haerendel, 1985; Baumjohann et al., 1985, 1986; Maynard et al., 1990; Matsui et al., 2005), and in the polar cap ionosphere (Ruohoniemi and Baker, 1998; Haaland et al., 2007). In the case of northward IMF, reconnection can take place between the IMF and already open polar cap field lines at high latitudes. In such cases, the polarity of the IMF $B_{x}$ component also plays a role. Positive (negative) $B_{x}$ favors lobe reconnection in the Southern (Northern) Hemisphere (Crooker, 1986).

Published by Copernicus Publications on behalf of the European Geosciences Union. 
The magnetotail lobes are bounded by the central plasma sheet and its boundary layer on one side, and the magnetopause with its adjacent boundary layer, often referred to as the plasma mantle (Rosenbauer et al., 1975), on the other side. The plasma mantle covers much of the high-latitude magnetosphere, extending poleward of the cusp region and is primarily populated with cold $(\simeq 100 \mathrm{eV})$, de-energized magnetosheath plasma with densities from $0.01-1 \mathrm{~cm}^{-3}$, and tailward flow velocities in the range $100-200 \mathrm{~km} \mathrm{~s}^{-1}$. Similarly, the plasma sheet boundary layer (PSBL), consists of hot plasma and has a density around $0.1-2 \mathrm{~cm}^{-3}$. The lobes are characterized by a very low particle density, typically 0.01 particles per $\mathrm{cm}^{-3}$ (e.g. Gosling et al., 1985), and a strong and steady magnetic field. Typical B-field values range from approximately 30-50 nT, somewhat dependent on geomagnetic activity (e.g. Caan et al., 1975). Tailward of approximately $10 R_{E}$, the magnetic field lines in the northern (southern) central lobe are almost parallel (anti parallel) to the $X_{\mathrm{GSE}}$ direction. The plasma transport is slow and steady towards the central plasma sheet.

The low particle density of the magnetotail lobes makes direct measurements with plasma instruments difficult. Most of the in-situ information from this region are therefore based on magnetic field instruments and E-field measurements from double probe instruments (see e.g. Svenes et al., 2008, and references therein)

In this paper we present a comprehensive survey of lobe convection as a function of IMF direction and magnitude as well as secondary effects reflected by various geomagnetic disturbance indicators. The survey is based on more than 340000 one-minute averages of the convection velocity obtained by the Cluster EDI instrument.

The paper is organized as follows: in Sect. 2, we present the EDI data set and auxillary data used for mapping and correlation studies. In Sect. 3 we describe the methods used to establish the concurrent solar wind condition and the procedure used for mapping of the EDI data. Section 4 presents the results, including correlations with the solar wind and disturbance indices and discusses possible physical implications. Section 5 gives an overall summary of the paper.

\section{Data}

The data set used in this study is similar to the data set used to study polar cap convection in Haaland et al. (2007) and Förster et al. (2007a), but covers a longer time interval, and is processed slightly differently. For convenience, we here briefly describe the data set.

\subsection{Cluster EDI data}

The European Space Agency (ESA) Cluster mission consists of four identical spacecraft flying in a tetrahedron-like formation. Cluster has a nearly $90^{\circ}$ inclination elliptical orbit with perigee around $4 R_{E}$ and apogee around $19 R_{E}$, and an orbital period of about $57 \mathrm{~h}$. Depending on season, Cluster traverses the magnetotail lobes, or field lines threading lobes during a substantial part of its orbit.

In this study, measurements of the plasma convection velocity are obtained from the Cluster Electron Drift Instrument (EDI). The basis of the electron-drift technique is the injection of two weak electron beams and their detection after one or more gyrations in the ambient magnetic field. Due to their cycloidal motion, the emitted electron beams return to the associated detectors only when fired in directions uniquely determined by the magnitude and direction of the plasma drift velocity. The drift velocity is computed either from the direction of the beams (via triangulation) or from the difference in their times-of-flight. More details about EDI can be found in Paschmann et al. (1997, 2001) and Quinn et al. (2001).

EDI measures the entire vector drift velocity, which, for a given magnetic field, is equivalent to the transverse electric field when the drift due to $\mathrm{B}$-field gradients is negligible. The E-field, which is given by $-\boldsymbol{V} \times \boldsymbol{B}$, thus includes any component along the spacecraft spin axis, whereas the double probe Electric Field and Wave Experiment (EFW) on board Cluster measures the electric field only in the spacecraft spin-plane. Another important advantage of EDI is its immunity to wake effects that can interfere with the doubleprobe measurements in regions of low plasma density (Eriksson et al., 2006). Also, EDI measurements are not affected by the low particle densities which limit the accuracy of the velocity moments derived from the CIS and PEACE plasma instruments.

The suitability of EDI for measurements in the polar cap region and the magnetotail lobes has been demonstrated in publications by e.g. Noda et al. (2003), Eriksson et al. (2006), Haaland et al. (2007) and Förster et al. (2007a). The large, relatively stable magnetic fields typically encountered by Cluster over the polar caps and in the lobes are a regime in which the EDI technique provides high accuracy with its gemoetric measurement technique.

EDI measurements are available for Cluster spacecraft 1 and 3 throughout the period February 2001 to June 2007, and until April 2004 for spacecraft 2. No EDI measurements are available from spacecraft 4 . Some operational constraints limit the availability of EDI data; EDI is an active experiment, and the emitted electron beam can under some circumstances modify the local plasma surroundings, and interfere with the wave measurements on Cluster. EDI is therefore operated with a duty-cycle that has been negotiated with the other experiments on Cluster. However, these restrictions primarily affect regions of low magnetic fields.

Since magnetic field lines above ionospheric heights can be considered as equipotential, we can utilize all EDI measurements along field lines threading the lobes, including measurements from sunward of the terminator. Figure 1 shows the distribution of the measurements as function of radial distance. The majority of measurements from the 


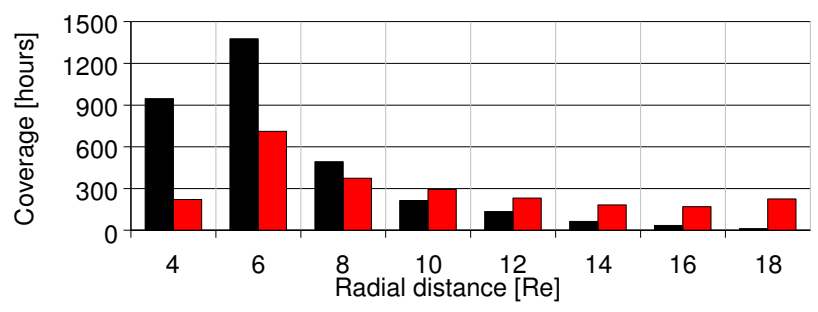

Fig. 1. Distribution of the EDI measurements versus geocentric radial distance. Black colors indicate Northern Hemisphere, red colors Southern Hemisphere. The majority of the EDI measurements in the Northern Hemisphere are taken at radial distances below $10 R_{E}$, whereas the Southern Hemisphere data set contains more measurements from distances beyond $10 R_{E}$.

northern lobe are taken from positions inside $10 R_{E}$, whereas the Southern Hemisphere data set contains relatively more measurements from distances beyond $10 R_{E}$. The reasons for this asymmetry is the Cluster orbit; the line of apsides of the orbit was initially equatorial, but is tilting more and more southward with time.

\subsection{Solar wind data and auxiliary data}

Measurements of the interplanetary magnetic field (IMF) and solar wind plasma data are obtained from the Advanced Composition Explorer (ACE) spacecraft. We have used data from the magnetic field instrument (MAG - see Smith et al., 1998) at 16-s resolution and the solar wind instrument (SWEPAM - see McComas et al., 1998) at 64-s resolution. MAG and SWEPAM data are resampled to one minute time resolution, thereafter time shifted to represent the IMF conditions at the frontside magnetopause.

The $D_{s t}$ index is used to study the correlation between convection and geomagnetic disturbance. The $D_{s t}$ index is a measure of the horizontal magnetic deflection on the Earth at equatorial latitudes. Negative deflections in $D_{s t}$ are mainly controlled by the Earth's ring current, though the solar wind pressure also contributes (e.g. Burton et al., 1975; O'Brien and McPherron, 2000). Positive deflections are usually caused by pressure enhancements in the solar wind which cause a displacement of magnetopause. The $D_{s t}$ index was provided in digital form by the World Data Center A, Kyoto.

The Auroral electrojet (AE) index, which reflects auroral geomagnetic activity, primarily associated with tail magnetic activity, was not continuously available for the entire period discussed here. We are therefore not able to discuss correlations with auroral activity or magnetospheric substorms.

\subsection{Data coverage}

Our dataset contains observations collected over a period of more than 6 years. During this time span, there were shorter
Table 1. Data coverage in hours of ACE and Cluster measurements used in the present study.

\begin{tabular}{lrrrrrr}
\hline \multirow{2}{*}{ Year } & \multirow{2}{*}{ Hours } & Valid & Stable & \multicolumn{3}{c}{ Mapped EDI vectors } \\
\cline { 5 - 7 } & & ACE & IMF & SC1 & SC2 1 & SC3 \\
\hline $2001^{2}$ & 8019 & 6845 & 4613 & 427 & 408 & 348 \\
2002 & 8760 & 7446 & 4632 & 593 & 409 & 681 \\
2003 & 8760 & 8294 & 3268 & 667 & 405 & 395 \\
2004 & 8784 & 7143 & 4181 & 626 & 60 & 524 \\
2005 & 8760 & 6450 & 4341 & 541 & - & 509 \\
2006 & 8760 & 6114 & 4252 & 379 & - & 411 \\
$2007^{3}$ & 4343 & 2785 & 2123 & 83 & - & 84 \\
Total & 56186 & 45077 & 27410 & 3319 & 1284 & 2955 \\
\hline
\end{tabular}

${ }^{1}$ Data from SC2 only until early April 2004.

2 Data for 2001 start in February.

3 Data only until 1 July 2007.

and longer periods where one or more of the data sources suffered from data gaps. Due to the Cluster high inclination orbit, EDI lobe data is only available during parts of the orbit. Also, in the early phase of the Cluster mission, there were telemetry constraints which prevented full data coverage. EDI is also subject to operational constraints to avoid interference with the wave and particle instruments onboard Cluster. There are also data gaps in the MAG and SWEPAM data from the ACE spacecraft, whereas the $D_{s t}$ and ASYM$\mathrm{H}$ indices are continuously available for the full period.

The treatment of data gaps is similar to those described in Haaland et al. (2007) and later also used by Förster et al. (2007a). Gaps in the solar wind observations from ACE of duration shorter than $10 \mathrm{~min}$ are linearly interpolated. For gaps longer than $10 \mathrm{~min}$, the solar wind conditions required for mapping cannot be established with reasonable confidence, and no further processing of the EDI data is done. However, the magnetic field model used for mapping (Tsyganenko, 2002a,b) requires solar wind history as an input parameter. The G1 and G2 factors of this model are based on the preceding $1 \mathrm{~h}$ history of the solar wind. If there is a gap within this period, the G1 and G2 factors are simply based on fewer samples. In the extreme case where the full preceding hour of data is missing, the G1 and G2 factors are both set to zero.

During the 77 months ( $\simeq 56000 \mathrm{~h}$ ) we have included in our study, ACE plasma and magnetic field data were available for approximately $45000 \mathrm{~h}$. Of this subset, slightly more than 27000 h satisfy our IMF stability criteria (see below). Highquality EDI data from Cluster SC1, SC2 and SC3 which could be mapped to the lobes were available for a total of almost $7600 \mathrm{~h}$ (453 667 one-minute averages). Details of the data coverage are given in Table 1.

Due to the Cluster orbit precession, the data coverage from EDI is correlated with season. For Northern Hemisphere late 


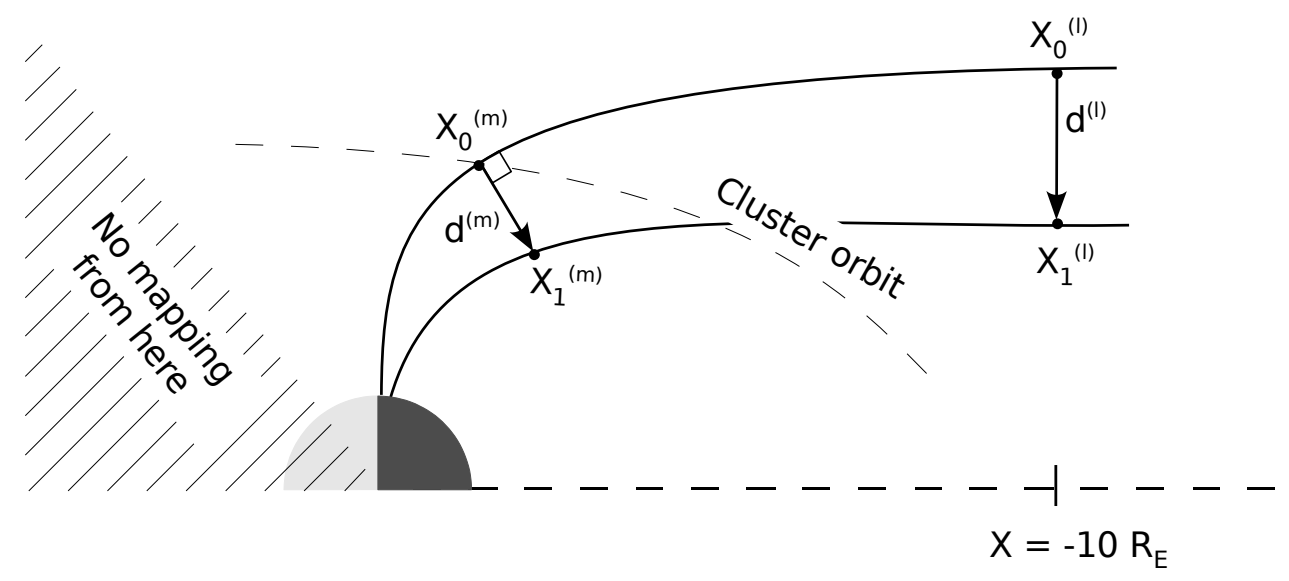

Fig. 2. Illustration of the mapping procedure. The vector $\boldsymbol{d}^{(m)}$, is along the convection velocity measured by EDI, and per definition perpendicular to the magnetic field at $x_{0}^{(m)}$. The vector $\boldsymbol{d}^{(m)}$ is projected along the field lines to the lobe at $X=-10 R_{E}$. The mapped vector $\boldsymbol{d}^{(l)}$ lies in the $Y Z_{\mathrm{GSM}}$ plane. The convection velocity in the lobe at $x_{0}^{(l)}$ is then $\boldsymbol{V}^{(l)}=\boldsymbol{V}^{(m)} \times d^{(l)} / d^{(m)}$.

summer (August to October), when Cluster's apogee is in the tail, we have more than $2100 \mathrm{~h}$ of data, whereas a little more than half as much data were collected during the months February to April. Due to the orientation of the Earth's tilt axis, the tail plasma sheet also has a similar seasonal dependence; during Northern Hemisphere summer, the nominal position of the plasma sheet is above the $\mathrm{XY}_{\mathrm{GSM}}$ plane. The dataset is therefore not suited for the study of seasonal effects.

\section{Method}

\subsection{Establishing the solar wind conditions}

The procedure to determine the concurrent IMF orientation is identical to the procedure described in Haaland et al. (2007) and later used by Förster et al. (2007a). Basically, it consists of time shifting the measurements from the ACE spacecraft, orbiting the L1 libration point, to the upstream magnetopause. For this purpose, we have applied the phase front propagation technique described in Weimer et al. (2003), although with a minor correction to the minimum variance calculation as suggested by Haaland et al. (2006), and later implemented in Weimer and King (2008). This technique takes the position of the solar wind monitor as well as the orientation of the IMF into account when calculating the time shift. For time intervals without any distinct IMF discontinuities, this method is believed to give more precise time shifts than simpler methods (Weimer and King, 2008, see also discussion in Mailyan et al., 2008). The time shifted IMF information is thereafter filtered with a bias filter so that only measurements which are stable within a $30 \mathrm{~min}$ interval are used. The interval is meant to take into account possible errors in the time shift estimate as well as some time to set up the mag- netospheric convection following IMF changes. Only EDI data within these stable IMF periods are used hereafter.

\subsection{Mapping the EDI data}

Under steady state conditions and assuming equipotential field lines, the convection at one location in space can in theory be mapped to any location along the field lines (e.g. Maynard et al., 1995; Hesse et al., 1997). Figure 2 illustrates the procedure to map the EDI vectors from the Cluster location to a $\mathrm{YZ}_{\mathrm{GSM}}$ plane at $X=-10 R_{E}$. This procedure is also basically the same as used in Haaland et al. (2007) and Förster et al. (2007a), except that we map outward into the magnetotail lobes rather than into the polar cap ionosphere, and use a larger data set.

The vector $\boldsymbol{V}^{(m)}$ is the convection velocity measured by EDI at Cluster, and $\boldsymbol{V}^{(l)}$ is the corresponding convection velocity in the lobe, i.e. our $\mathrm{YZ}_{\mathrm{GSM}}$ plane at $X=-10 R_{E}$ (we hereafter use superscripts $m$ to indicate the position of Cluster and $l$ to the indicate a the mapped position in the lobe). The magnetic field lines are assumed to be equipotential.

Using the Tsyganenko T2001 magnetic field model (Tsyganenko, 2002a,b) the Cluster position, $x_{0}^{(m)}$, is mapped along the field line to the position $x_{0}^{(l)}$ in the lobe. In addition, the point $x_{1}^{(m)}$, which is displaced a distance $\boldsymbol{d}^{(m)}$ along the measured convection velocity, $\boldsymbol{V}^{(m)}$, is mapped to the position $x_{1}^{(l)}$, also at $X=-10 R_{E}$ in the lobe. We have chosen the scaling so that the corresponding distance $\boldsymbol{d}^{(l)}=x_{1}^{(l)}-x_{0}^{(l)}$, is $1 R_{E}$. Since the magnetic field diverge or converge (depending on whether we map from a high magnetic field to a lower field or vice versa), $d^{(m)}$ is then given by $d^{(m)}=\sqrt{B^{(l)} / B^{(m)}} \times 1 R_{E}$. The choice of $1 R_{E}$ distance between mapped field lines in the lobe was found to be a reasonable compromise between uncertainties and distortion in 


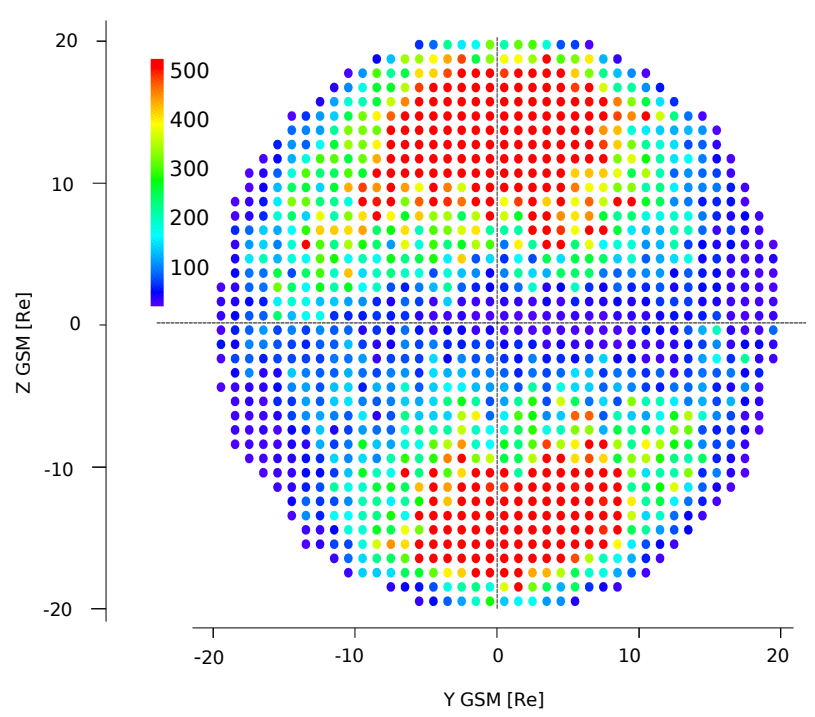

Fig. 3. Spatial coverage. Approximately 340000 data points (corresponding to more than $5700 \mathrm{~h}$ of measurements) have been mapped into $1 \times 1 R_{E}$ bins at a plane $10 R_{E}$ downtail. The colors of the symbols indicate the number of mapped EDI vectors in each bin. The view is from the Earth, looking tailward.

the mapping. The convection velocity in the lobe at $x_{0}^{(l)}$ is then $\boldsymbol{V}^{(l)}=\boldsymbol{V}^{(m)} \times d^{(l)} / d^{(m)}$.

Note that this mapping procedure projects the results into the $X=-10 R_{E}$ plane, and does not take any X-component of the convection into account. However, since the magnetic field in the central lobes is predominantly in the $\pm X_{\mathrm{GSE}}$ direction, the average $V x$ convection is negligible at $X=-10 R_{E}$.

We also tested mapping to a plane at $X=-15 R_{E}$, and also mapping using a Solar Magnetic (SM) coordinate system, but the overall results were similar. One motivation for using $X=-10 R_{E}$ and a GSM coordinate system was to facilitate comparison with the results of Noda et al. (2003). Also, although technically possible, an $X=-15 R_{E}$ limit is strictly beyond the validity region of the T2001 magnetic field model.

As shown in Fig. 1, most of the data are obtained from geocentric distances around $4-8 R_{E}$. The mapping distances (i.e. the length along a field line from the position of Cluster to our plane at $X=-10 R_{E}$ ) are therefore typically around 2-6 $R_{E}$. Since Cluster also traverses the $X=-10 R_{E}$ plane during some orbits, and no mapping is required, parts of the data can be regarded as direct, in-situ measurements.

\section{Results}

In the following, we will discuss a filtered data set; Mapped vectors with velocities of more than $50 \mathrm{~km} \mathrm{~s}^{-1}$ have been filtered out, as theses can safely be regarded as outliers. Also,
Table 2. Summary of convection velocities, Vz, Vy, and the cross tail potential, $\mathrm{U}_{C T}$ for various IMF directions. The rightmost column shows the corresponding average of the full data set without any IMF sorting.

\begin{tabular}{|c|c|c|c|c|c|}
\hline $\begin{array}{l}\text { Northern } \\
\text { lobe }\end{array}$ & $B_{z^{+}}$ & $B_{y}+$ & $\begin{array}{l}B_{z}- \\
\square\end{array}$ & $B_{y-}$ & All \\
\hline Num records & 13985 & 29832 & 18120 & 32249 & 94186 \\
\hline $\mathrm{Vz}\left[\mathrm{km} \mathrm{s}^{-1}\right]$ & -2.0 & -7.2 & -10.2 & -7.3 & -7.0 \\
\hline $\mathrm{Vy}\left[\mathrm{km} \mathrm{s}^{-1}\right]$ & 0.2 & 6.5 & 0.0 & -7.7 & -0.5 \\
\hline $\mathrm{U}_{C T}[\mathrm{kV}]$ & 21.4 & 41.6 & 61.6 & 42.2 & 41.2 \\
\hline
\end{tabular}

\begin{tabular}{|c|c|c|c|c|c|}
\hline $\begin{array}{l}\text { Southern } \\
\text { lobe }\end{array}$ & $\stackrel{B_{z}^{+}}{\odot}$ & $\begin{array}{l}B_{y}+ \\
\bigcirc\end{array}$ & $\begin{array}{l}B_{z}- \\
\square\end{array}$ & $B^{B^{-}}$ & All \\
\hline Num records & 10710 & 30986 & 14776 & 25963 & 82435 \\
\hline $\mathrm{Vz}\left[\mathrm{km} \mathrm{s}^{-1}\right]$ & 2.9 & 8.7 & 12.8 & 7.0 & 8.1 \\
\hline $\mathrm{Vy}\left[\mathrm{km} \mathrm{s}^{-1}\right]$ & -0.3 & -4.8 & 5.4 & 10.7 & 2.5 \\
\hline $\mathrm{U}_{C T}[\mathrm{kV}]$ & 19.8 & 33.7 & 55.1 & 32.2 & 39.4 \\
\hline
\end{tabular}

we focus our study to a circular area with a radius of $20 R_{E}$ to avoid regions at or near the magnetopause. This filtered data set contains a total of 342544 records (approximately 5709 h). Figure 3 shows the spatial coverage of our data set. For the central lobes, where the magnetic field is strong and fairly stable, and the background electron density is low, EDI operates nearly continuously, and the coverage is excellent.

\subsection{Convection patterns in the lobes}

Figure 4 shows the convection patterns for the four main directions of the interplanetary magnetic field. The colored dots represent the center of each $1 \times 1 R_{E}$ bin, and the lines out from each dot show the average convection direction and strength in that particular bin. Colors indicate the normalized total variance (see Sect. 4.2 below). Only bins containing 2 or more measurements are shown. The blue rectangular areas in the northern and southern lobe are used to calculate the characteristic averages listed in Table 2 (see also Sect. 4.3).

The upper panels of Fig. 4 show the convection pattern for northward (left panel) and southward (right panel) interplanetary field. For northward IMF, the convection is slow and with an unsystematic pattern. Except for some bins close to the magnetopause, most of the bins have convection velocities of maximum a few $\mathrm{km} \mathrm{s}^{-1}$. This is expected, as there is little or no reconnection on the dayside magnetopause in such cases. The small average $V z$ components seen in Table 2 nevertheless indicate a slow transport towards the plasma sheet. This is most likely a flywheel effect (see e.g. Ridley et al., 2003, and references therein), i.e. the magnetospheric circulation does not react instantaneously on a northward turning of the IMF. However, since our data set does not consist of continous time series, we are not able to investigate 

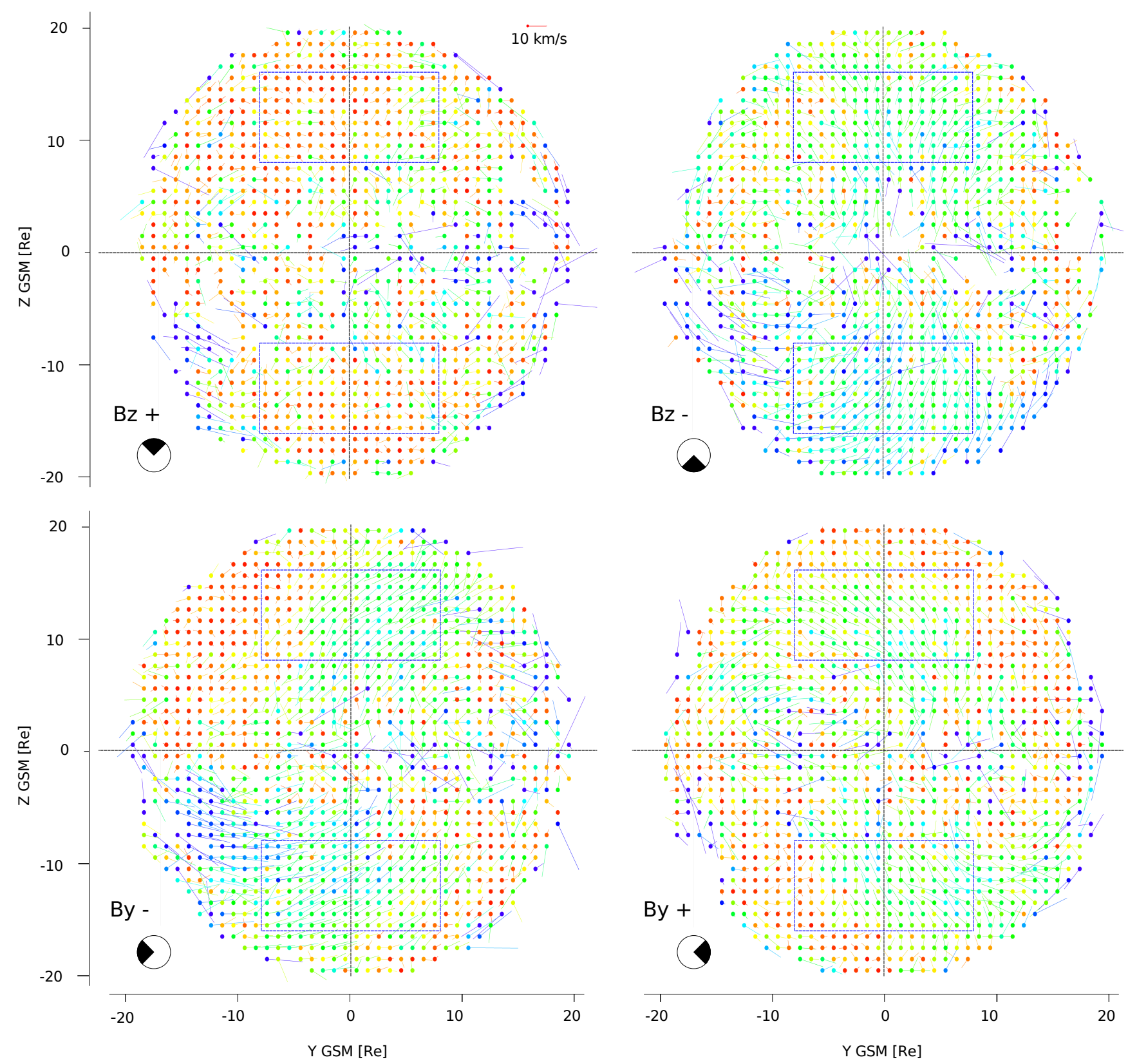

$\begin{array}{lllllllllll}0 & 0.1 & 0.2 & 0.3 & 0.4 & \begin{array}{l}0.5 \\ \text { Total variance }\end{array} & 0.6 & 0.8 & 0.9 & 1.0\end{array}$

Fig. 4. Convection patterns in the lobe at $X=-10 R_{E}$ as function of the IMF direction. Sector symbols in the lower left corner of each panel indicate the IMF clock angle sector, and the colors and color bar indicate the normalized total variance for each bin. The view is from the Earth, looking tailward. Blue boxes in the northern and southern lobe are two reference regions used for calculation of characteristic averages and correlations between convection and various driver parameters. Average convection velocities, calculated cross-tail potential drop and the number of records within these regions are given in Table 2.

any temporal effects of the convection patterns. Ionospheric convection during northward IMF is discussed in more detail in the a companion paper by Förster et al. (2007b).
For southward IMF, there is a strong and more laminar convection towards the central plasma sheet with convection velocities of more than $10 \mathrm{~km} \mathrm{~s}^{-1}$. In our data set, the 
southern lobe values are slightly higher, but we do not know if this is real, or an artifact of the unequal radial distribution of the northern and southern measurements (see Fig. 1).

The lower panels of Fig. 4 shows the $B_{y}$ dependence of the convection. Due to the typical Parker spiral orientation of the IMF, the coverage is significantly better here. The convection now has a significant dawn-dusk component. For IMF $B_{y}$ positive (lower right panel), the convection is duskward in the northern lobe, and dawnward in the southern lobe. The opposite is the case for negative IMF $B_{y}$, but the results are not mirror symmetric, and the convection variations seem to be modulated on an overall persistent but weak duskward $V y$ convection of a about $1 \mathrm{~km} \mathrm{~s}^{-1}$. This asymmetry is discussed further in the next section.

Except from the northward IMF case, the convection patterns seem to form some large scale vortices. We believe that these reflect the convection cells seen in the ionosphere (see Fig. 7 in Haaland et al., 2007). This is an illustration that magnetospheric convection is not simply the result of the solar wind input or processes at the magnetospheric boundaries or the magnetotail, but that it is also partly controlled by the drag of the ionosphere (e.g. Wolf, 1970; Coroniti and Kennel, 1973; Ridley et al., 2003).

To double check the results, we also tried to estimate the cross tail potential drop, $U_{C T}$. This is done by first calculating the convection electric field in each bin (from $\boldsymbol{E}=-\boldsymbol{V} \times \boldsymbol{B}$, where the B-field is the field given by the magnetic field model for that location). Thereafter, we integrate this electric field across the tail along the $\mathrm{Z}_{\mathrm{GSM}}= \pm 5 R_{E}$ lines in the northern and southern lobe. The choice of $5 R_{E}$ was done to avoid the central plasma sheet (including the seasonal motion of the plasma sheet) where B-field fluctuations and higher background plasma densities limit the EDI operation and the coverage is very poor.

In theory, the cross tail potential should be equal to the cross-polar cap potential for equipotential magnetic field lines. The result, listed in Table 2 shows the expected dependence of IMF direction, although with lower absolute values than the polar cap potentials obtained by e.g. Papitashvili and Rich (2002); Weimer (2005), but comparable to the polar cap potentials obtained by Ruohoniemi and Greenwald (2005) and Haaland et al. (2007).

\subsection{Convection variability}

To assess the uncertainty of the results, we also calculate two variances for each bin. The approach is similar to the procedure used by Förster et al. (2007a) with EDI data, and also used by the Cluster magnetic field experiment (FGM - see Balogh et al., 2001) to quantify statistical errors of vector quantities. The two variances are expressed as:

$\sigma_{\mathrm{mag}}^{2}=\frac{\left\langle|\boldsymbol{V}|^{2}\right\rangle-\langle|\boldsymbol{V}|\rangle^{2}}{\left\langle|\boldsymbol{V}|^{2}\right\rangle}$ $\sigma_{\text {total }}^{2}=\frac{\left\langle|\boldsymbol{V}|^{2}\right\rangle-|\langle\boldsymbol{V}\rangle|^{2}}{\left\langle|\boldsymbol{V}|^{2}\right\rangle}$

where $\boldsymbol{V}=\boldsymbol{V}^{(l)}$ is the convection velocity vector in the lobe, and $\langle.$.$\rangle denotes an average over all vectors in that bin. Both$ variances are normalized and take values between 0 and 1 .

The first quantity, $\sigma_{\mathrm{mag}}^{2}$, is the normalized variance of the magnitude of the convection velocity, and describes the deviation of the convection magnitude from its average value.

More relevant four our study is the second variance, $\sigma_{\text {total }}^{2}$, which is the normalized variance of the total convection velocity vector. This variance is indicated by colors in Fig. 4 . Zero total variance (blue color in Fig. 4) indicates steady pointing vectors and thus a well defined convection direction. Correspondingly, values of $\sigma_{\text {total }}^{2}$ close to unity (red color) indicate that the convection vectors in that bin are distributed in all directions.

For northward IMF, $\sigma_{\text {total }}^{2}$ approaches unity in many bins, whereas for southward and $B_{y}$ dominated IMF directions the $\sigma_{\text {total }}^{2}$ is in general much lower. In particular, there is a well defined convection direction in the Northern Hemisphere dawn and Southern Hemisphere dusk region for positive IMF $B_{y}$ and vice versa for negative IMF $B_{y}$. A similar asymmetry in the ionospheric convection was also found by Förster et al. (2007a).

\subsection{Correlations}

To quantify the influence of the solar wind, IMF direction and geomagnetic disturbances, we examine the average convection within the above mentioned reference area in our $X=-10 R_{E}$ projection plane. A similar approach was used by Noda et al. (2003), although they used a slightly different area and had a much smaller data set available. They were therefore not able to address the dependence of solar wind and IMF condition in so much detail.

The reference areas selected are indicated by the blue rectangles in Fig. 4. We perform the calculation for northern and southern lobe separately. The two regions extend from $\mathrm{Y}_{\mathrm{GSM}}=-8 R_{E}$ to $+8 R_{E}$ and $\left|Z_{\mathrm{GSM}}\right|=8$ to $16 R_{E}$, and contain 128 bins. The location and size was chosen so that it covers the central lobes, but avoids regions close to the central plasma sheet and the magnetopause. During extreme geomagnetic conditions, the magnetopause may be compressed, or the central plasma sheet may be displaced so that it is located inside our reference area. The contamination from such events are minimal though, and we do not think such conditions skew our results.

For each of the driver parameters: IMF direction, IMF magnitude and solar wind dynamic pressure as well as the $D_{s t}$ index, we calculate the average convection velocities within the reference area for subsequent ranges of the driver parameter. The binning of the driver parameters is a compromise between adequate resolution and sufficient data coverage. For most of the ranges, the averages are based on several thousand records, and the statistical uncertainty is negligible. 
However, for extreme values of some of the driver parameters, the coverage (and thus the statistical confidence) is sometimes poor, and results from these ranges should thus be considered with caution. When interpreting these results, one should also have in mind that the driver parameters are partly mutually correlated (see e.g. Table 1 of Förster et al., 2007a); for example, the effects reflected by the $D_{s t}$ index ultimately driven by the solar wind.

The results are given in Fig. 5. The left panels show histograms of the Z-component of the convection for different ranges of the driver parameters. $V z$ is oppositely directed in Northern and Southern Hemisphere. To be able to use the same scale and thus facilitate easier comparison, we have inverted the $V z$ values for the Northern Hemisphere lobe. The right panels of Fig. 5 show the corresponding $V y$ convections. Also shown here as lines is the data coverage. A $100 \%$ coverage indicate that all of the 128 bins within our reference area have measurements.

In the following, we discuss each correlation in some detail.

\subsubsection{IMF direction}

The overall response to the IMF direction (here represented by the solar wind clock angle) was already discussed in the previous section (Fig. 4). To further quantify the effect of the IMF direction, we use a similar approach as Förster et al. (2007a), and divide the IMF clock angle into 8 sectors, each 45 degrees wide. For each such sector, we then calculate the average convection velocity within our reference area. The results show a clear correlation; the convection is very low during purely northward IMF, and maximizes for southward IMF. Similarly, clock angles close to $\pm 90^{\circ}$ cause strong convection in the dawn-dusk direction with opposite directions in the northern and southern lobes.

This asymmetry can be understood from Fig. 6, which provides a schematic illustration of dayside magnetopause reconnection and the corresponding plasma entry into the tail during a southward, but strongly $B_{y}$ dominated IMF. For a positive IMF $B_{y}$, the reconnected field lines in the Northern Hemisphere are dragged tailward on the dawnside, and the plasma entry takes place along the dawn side of the magnetopause (e.g. Gosling et al., 1984, 1985; Siscoe and Sanchez, 1987; Sibeck et al., 1999; Hasegawa et al., 2002a,b). $B_{y}$ the same token, the plasma entry in the Southern Hemisphere will take place on the dusk flank for this IMF configuration. Inside the magnetosphere, the asymmetric entry will cause a duskward component of the convection in the northern lobe and a dawnward convection in the southern lobe. For a negative IMF $B_{y}$ component, this scenario is basically mirrored. Observations also indicate that the whole plasma sheet eventually tilts as a result of the asymmetric plasma entry (Russell and Brody, 1967; Sibeck et al., 1985; Hammond et al., 1994).

An interesting result, which was also noted by Noda et al. (2003) is the apparent presence of a background duskward convection, most prominent in the southern lobe, but also apparent in the northern lobe. We believe that this persistent $V y$ convection is the magnetospheric manifestation of the day-night gradient in the ionospheric conductivity (Atkinson and Hutchison, 1978; Tanaka, 2001), and also pointed out in the EDI dataset mapped to the ionosphere (Haaland et al., 2007; Förster et al., 2007a). This asymmetry causes a rotation and break of IMF $B_{y}$ symmetry in the ionospheric convection. Since most of the lobe regions we are discussing here are threaded by magnetic field lines with footpoints in the high latitude nightside ionosphere, this asymmetry is also reflected in the lobes.

For each range of IMF direction, we also calculated the cross tail potential using the method described above. The calculated potentials are shown as solid lines in the upper left panel of Fig. 5. As expected, the minimum potential can be found for northward or nearly northward IMF, whereas the potential maximizes for southward IMF. As pointed out, the method is not terribly accurate, and for some of the IMF directions, the coverage along our $Z= \pm 5 R_{E}$ integration lines is marginal. The seemingly low potential in southern lobe for southward directed IMF is an artifact of this marginal coverage.

\subsubsection{IMF magnitude}

To study the influence of the IMF magnitude, we check the correlation between the magnitude of IMF in the YZ plane; $\left|B_{y z}^{\mathrm{MF}}\right|=\sqrt{B_{z}^{2}+B_{y}^{2}}$ and the convection. The IMF x component is not included here to, as it is believed to have little or no influence on the dayside reconnection rate. The plots indicate a weak positive correlation with $V z$, and a persistent positive (i.e. duskward) convection component in the Southern Hemisphere and, for high values of $\left|B_{y z}^{\mathrm{MF}}\right|$, a dawnward component in the northern lobe. Due to the low data coverage for extreme values of $\left|B_{y z}^{\mathrm{MF}}\right|$, the corresponding potential calculation does not make sense here.

The correlation with $\left|B_{y z}^{\mathbb{M F}}\right|$, also depends on the IMF direction. Due to insufficient statistics, we were not able to study the dependence for all directions of the IMF, but a separation into northward $\left(B_{z} \geq 0\right)$ and southward IMF $\left(B_{z} \leq 0\right)$ revealed that the correlation seen in Fig. 5b) is much more pronounced for the data set with southward IMF.

\subsubsection{Solar wind dynamic pressure}

The solar wind dynamic pressure, $P_{\mathrm{dyn}}=n m_{i} v_{s w}^{2}$, is a function of solar wind velocity, $v_{s w}$, and density only ( $n$ is the number density, $m_{i}$ is the proton mass), and is thus intrinsically independent of IMF direction. The $\mathrm{Vz}$ convection is highest for low pressure (first bin $-0-1 \mathrm{nPa}$ ). A similar result was also reported by (Förster et al., 2007a), but we do not have an explanation for this behavior. There is weak anti-correlation up to about $4 \mathrm{nPa}$ for the southern lobe and $6 \mathrm{nPa}$ for the northern lobe. For pressures below $4 \mathrm{nPa}$, the 

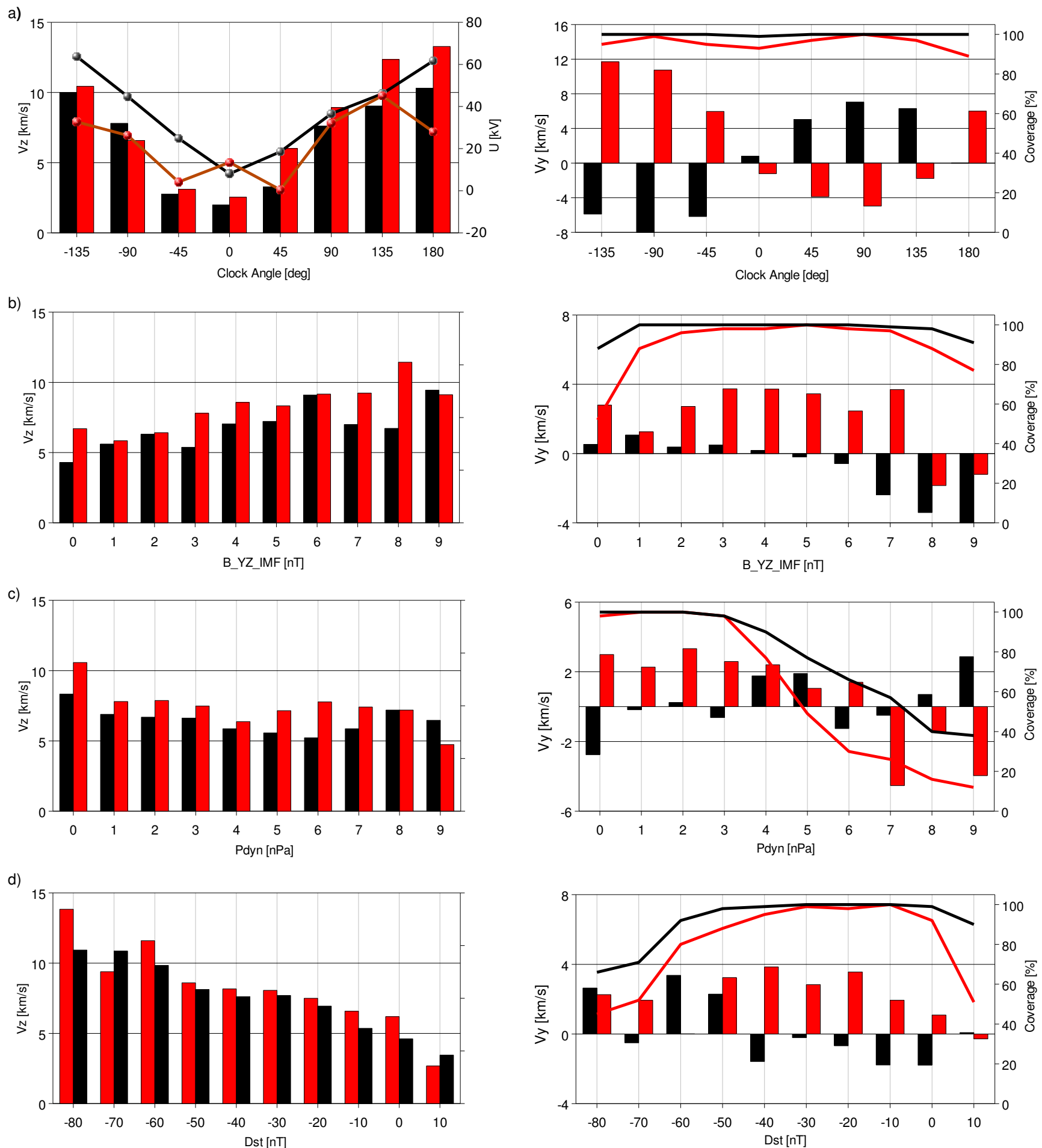

Fig. 5. Correlation between convection and solar wind and disturbance parameters. Left panels: Average convection velocities $V z$ (bars) and calculated cross tail potential (solid lines, top left panel only). Right panels: Corresponding Vy convection (bars) and data coverage (solid lines). Black (red) color indicates Northern (Southern) Hemisphere. For the upper panel, the numbers along the horizontal axis show the center of the clock angle sector, i.e. 0 means the average obtained from the $45^{\circ}$ clock angle sector ranging from from -22.5 to +22.5 degrees. For the other parameters, the numbers indicate the beginning of the range. 

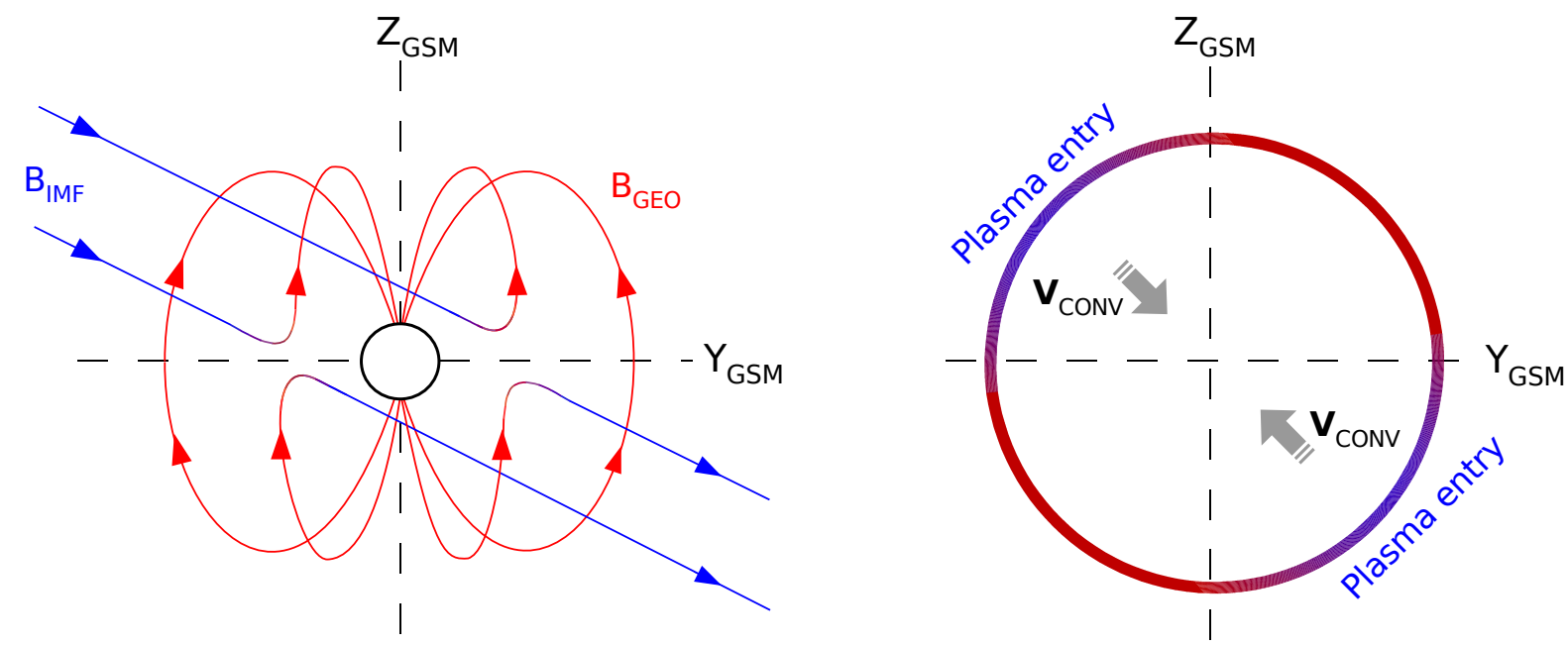

Fig. 6. Schematic illustration of the dayside reconnection (left) and corresponding plasma entry and convection towards the tail plasma sheet (right) for a southward IMF with a strong positive $B_{y}$ component. The view is from the Sun and looking tailward. The geomagnetic field (red lines) reconnects to the IMF (blue lines) and are dragged tailward along the flanks. For this IMF configuration, the plasma entry takes place along the the blue marked regions in the northern dawn and southern dusk flank regions.

coverage is excellent, and the persistent duskward convection component is apparent in the southern lobe.

Since our dataset does not consist of continuous time series, we are not able to investigate the direct response to time variances such as pressure pulses or sudden reductions in the dynamic pressure.

\subsection{4 $D_{s t}$ index}

The $D_{s t}$ index is a measure of the horizontal magnetic deflection on the Earth at equatorial latitudes. Negative deflections are mainly controlled by the Earth's ring current, though there is some contribution from the solar wind pressure (e.g. Burton et al., 1975; O'Brien and McPherron, 2000) and also from tail activity like bursty bulk flows or substorm activity (e.g. Baumjohann et al., 1996; Baker et al., 2001). Energization of the ring current is usually very fast, whereas loss processes such as charge exchange, pitch angle scattering etc. have much longer time scales (e.g. Cowley, 1977; Daglis et al., 1999).

Typically, the ring current increases (thus creating a negative deflection of $D_{s t}$ ) during periods of increased cross-tail electric field, which is typically caused by enhanced dayside reconnection. The clear correlation between $\mathrm{Vz}$ and $D_{s t}$ apparent from the plot seems to confirm this argument.

\section{Summary}

Based on more than $5700 \mathrm{~h}$ of measurements from the Cluster EDI experiment we have investigated the overall convection in the magnetotail tail lobes. The EDI measurements, obtained at geocentric distances between 4 and $19 R_{E}$, are mapped into a $\mathrm{YZ}_{\mathrm{GSM}}$ plane at $X=-10 R_{E}$ downtail, and sorted according to the direction of the interplanetary magnetic field.

- The convection velocity and direction depend strongly on the IMF direction. For purely northward IMF (clock angles in the range $0 \pm 22.5^{\circ}$ ), the convection is almost stagnant. For southward IMF (clock angle = $180 \pm 22.5^{\circ}$ ), there is a strong convection towards the plasma sheet. A southward IMF with a strong $B_{y}$ component causes a rotation of the plasma entry regions towards the dusk/dawn flanks and a corresponding dawndusk component of the convection in the northern lobe, and an oppositely directed dawn-dusk component in the southern lobe.

- The calculated cross-tail potential is comparable to the ionospheric polar cap potential and shows the same IMF direction dependence as the polar cap potential drop.

- The convection velocity is correlated with the IMF magnitude, and appears to be weakly anti-correlated to the solar wind dynamic pressure.

- There is a positive correlation between the $D_{s t}$ index (which reflects ring current enhancements) and the convection velocity. This suggests that a major part of the ring current enhancement is directly driven by dayside reconnection.

- There seems to be a persistent duskward component of the convection with an average velocity of around $1.5 \mathrm{~km} \mathrm{~s}^{-1}$. 
- Except for northward IMF, the convection patterns show signatures of large scale vortices. These are most likely manifestations of the ionospheric convection.

Acknowledgements. Work at the Max-Planck Institut was supported by Deutsches Zentrum für Luft- und Raumfahrt (DLR). Work at GeoForschungsZentrum (GFZ) Potsdam was supported by Deutsche Forschungsgemeinschaft (DFG). Research at the University of Bergen and University of Oslo was supported by the Norwegian Research Council. Work by US investigators was supported in part by NASA grant NNG04GA46G. Parts of the data analysis were done with the QSAS science analysis system provided by the UK Cluster Science Centre (Imperial College London and Queen Mary, University of London) supported by The Science and Technology Facilities Council (STFC), United Kingdom. We thank the ACE SWEPAM and MAG instrument teams and the ACE Science Center for providing the ACE data, and the World Data Center for Geomagnetism, Kyoto, for providing the $D_{s t}$ and ASYM-H indices. We thank M. Chutter for his support of EDI data analysis and processing, and G. Leistner for providing the averaged EDI data.

Topical Editor R. Nakamura thanks W. Baumjohann and C.P. Wang for their help in evaluating this paper.

\section{References}

Atkinson, G. and Hutchison, D.: Effect of the day night ionospheric conductivity gradient on polar cap convective flow, J. Geophys. Res., 83, 725-729, 1978.

Baker, D. N., Turner, N. E., and Pulkkinen, T. I.: Energy transport and dissipation in the magnetosphere during geomagnetic storms, J. Atmos. Terr. Phys., 63, 421-429, 2001.

Balogh, A., Carr, C. M., Acuña, M. H., Dunlop, M. W., Beek, T. J., Brown, P., Fornacon, K. H., Georgescu, E., Glassmeier, K. H., Harris, J., Musmann, G., Oddy, T., and Schwingenschuh, K.: The Cluster Magnetic Field Investigation: Overview of in-flight performance and initial results, Ann. Geophys., 19, 1207-1217, 2001 , http://www.ann-geophys.net/19/1207/2001/.

Baumjohann, W. and Haerendel, G.: Magnetospheric convection observed between 0600 and 2100 LT Solar wind and IMF dependence, J. Geophys. Res., 90, 6370-6378, 1985.

Baumjohann, W., Haerendel, G., and Melzner, F.: Magnetospheric convection observed between 0600 and 2100 LT Variations with Kp, J. Geophys. Res., 90, 393-398, 1985.

Baumjohann, W., Nakamura, R., and Haerendel, G.: Dayside equatorial-plane convection and IMF sector structure, J. Geophys. Res., 91, 4557-4560, 1986.

Baumjohann, W., Kamide, Y., and Nakamura, R.: Storms, substroms and the near-Earth tail, J. Geophys. Geomagn., 48, $177-$ 185, 1996.

Burton, R. K., McPherron, R. L., and Russell, C. T.: An empirical relationship between interplanetary conditions and $D_{s t}$, J. Geophys. Res., 80, 4204-4214, 1975.

Caan, M. N., McPherron, R. L., and Russell, C. T.: Substorm and interplanetary magnetic field effects on the geomagnetic tail lobes, J. Geophys. Res., 80, 191-194, 1975.

Coroniti, F. V. and Kennel, C. F.: Can the ionosphere regulate magnetospheric convection?, J. Geophys. Res., 78, 2837-2851, 1973.
Cowley, S. W. H.: Pitch angle dependence of the charge-exchange lifetime of ring current ions, Planet. Space Sci., 25, 385-393, doi:10.1016/0032-0633(77)90054-X, 1977.

Cowley, S. W. H., Morelli, J. P., and Lockwood, M.: Dependence of convection flows and particle precipitation in the high-latitude dayside ionosphere on the $\mathrm{X}$ and $\mathrm{Y}$ components of the interplanetary magnetic field, J. Geophys. Res., 96, 5557-5564, 1991.

Crooker, N. U.: An evolution of antiparallel merging, Geophys. Res. Lett., 13, 1063-1066, 1986.

Daglis, I. A., Thorne, R. M., Baumjohann, W., and Orsini, S.: The terrestrial ring current: Origin, formation, and decay, Rev. Geophys., 37, 407-438, doi:10.1029/1999RG900009, 1999.

Dungey, J. R.: Interplanetary magnetic field and the auroral zones, Phys. Rev. Lett., 6, 47-48, 1961.

Eriksson, A. I., André, M., Klecker, B., Laakso, H., Lindqvist, P.-A., Mozer, F., Paschmann, G., Pedersen, A., Quinn, J., Torbert, R., Torkar, K., and Vaith., H.: Electric field measurements on Cluster: comparing the double-probe and electron drift techniques., Ann. Geophys., 24, 275-289, 2006, http://www.ann-geophys.net/24/275/2006/.

Förster, M., Paschmann, G., Haaland, S. E., Quinn, J. M., Torbert, R. B., Vaith, H., and Kletzing, C. A.: High-Latitude Plasma Convection form Cluster EDI: Variances and Solar Wind Correlation, Ann. Geophys., 25, 1691-1707, 2007a, http://www.ann-geophys.net/25/1691/2007/.

Förster, M. Haaland, S. E., Paschmann, G., Quinn, J. M., Torbert, R. B., Vaith, H., and Kletzing, C. A.: High-latitude plasma convection from Cluster EDI: variances and solar wind correlations, Ann. Geophys., 25, 1691-1707, 2007b, http://www.ann-geophys.net/25/1691/2007/.

Gosling, J. T., Baker, D. N., Bame, S. J., Hones Jr., E. W., McComas, D. J., Zwickl, R. D., Slavin, J. A., Smith, E. J., and Tsurutani, B. T.: Plasma entry into the distant tail lobes - ISEE-3, Geophys. Res. Lett., 11, 1078-1081, 1984.

Gosling, J. T., Baker, D. N., Bame, S. J., Feldman, W. C., and Smith, E. J.: North-south and dawn-dusk plasma asymmetries in the distant tail lobes - ISEE 3, J. Geophys. Res., 90, 6354-6360, 1985.

Haaland, S., Paschmann, G., and Sonnerup, B. U. Ö.: Comment on "A new interpretation of Weimer et al.'s solar wind propagation delay technique" by Bargatze et al., J. Geophys. Res. (Space Physics), 111, 6102-6106, doi:10.1029/2005JA011376, 2006.

Haaland, S., Förster, M., and Paschmann, G.: High-latitude plasma convection from Cluster EDI measurements: method and IMFdependence, Ann. Geophys., 25, 239-253, 2007, http://www.ann-geophys.net/25/239/2007/.

Hammond, C. M., Kivelson, M. G., and Walker, R. J.: Imaging the effect of dipole tilt on magetotail boundaries, J. Geophys. Res., 99, 6079-6092, 1994.

Hasegawa, H., Maezawa, K., Mukai, T., and Saito, Y.: Plasma entry across the distant tail magnetopause 1. Global properties and IMF dependence, J. Geophys. Res., 107, 1063, doi: 10.1029/2001JA900139, 2002a.

Hasegawa, H., Maezawa, K., Mukai, T., and Saito, Y.: Plasma entry across the distant tail magnetopause 2 . Comparison between MHD theory and observation, J. Geophys. Res., 107, 1073, doi: 10.1029/2001JA900138, 2002b.

Hesse, M., Birn, J., and Hoffman, R. A.: On the mapping of ionospheric convection into the magnetosphere, J. Geophys. Res., 102, 9543-9552, doi:10.1029/96JA03999, 1997. 
Mailyan, B., Munteanu, C., and Haaland, S.: What is the best method to calculate the solar wind propagation delay?, Ann. Geophys., 26, in press, 2008.

Matsui, H., Quinn, J. M., Torbert, R. B., Jordanova, V. K., PuhlQuinn, P. A., and Paschmann, G.: IMF BY and the seasonal dependences of the electric field in the inner magnetosphere, Ann. Geophys., 23, 2671-2678, 2005, http://www.ann-geophys.net/23/2671/2005/.

Maynard, N. C., Sojka, J. J., Schunk, R. W., Heppner, J. P., and Brace, L. H.: A Test of convection models for IMF $B_{z}$ North, Planet. Space Sci., 38, 1077-1089, doi:10.1016/0032-0633(90) 90017-K, 1990.

Maynard, N. C., Denig, W. F., and Burke, W. J.: Mapping ionospheric convection patterns to the magnetosphere, J. Geophys. Res., 100, 1713-1721, 1995.

McComas, D. J., Bame, S. J., Barker, P., Feldman, W. C., Phillips, J. L., Riley, P., and Griffee, J. W.: Solar Wind Electron Proton Alpha Monitor (SWEPAM) for the Advanced Composition Explorer, Space Sci. Rev., 86, 563-612, 1998.

Noda, H., Baumjohann, W., Nakamura, R., Torkar, K., Paschmann, G., Vaith, H., Puhl-Quinn, P., Förster, M., Torbert, R., and Quinn, J. M.: Tail lobe convection observed by Cluster/EDI, J. Geophys. Res., 108, 1288, doi:10.1029/2002JA009669, 2003.

O'Brien, T. P. and McPherron, R. L.: An empirical phase space analysis of ring current dynamics: Solar wind control of injection and decay, J. Geophys. Res., 105, 7707-7720, doi:10.1029/ 1998JA000437, 2000.

Papitashvili, V. O. and Rich, F. J.: High-latitude ionospheric convection models derived from Defense Meteorological Satellite Program ion drift observations and parameterized by the interplanetary magnetic field strength and direction, J. Geophys. Res., 107, 1198, doi:10.1029/2001JA000264, 2002.

Paschmann, G., Melzner, F., Frenzel, R., Vaith, H., Parigger, P., Pagel, U., Bauer, O. H., Haerendel, G., Baumjohann, W., Scopke, N., Torbert, R. B., Briggs, B., Chan, J., Lynch, K., Morey, K., Quinn, J. M., Simpson, D., Young, C., McIlwain, C. E., Fillius, W., Kerr, S. S., Mahieu, R., and Whipple, E. C.: The Electron Drift Instrument for Cluster, Space Sci. Rev., 79, 233-269, 1997.

Paschmann, G., Quinn, J. M., Torbert, R. B., Vaith, H., McIlwain, C. E., Haerendel, G., Bauer, O. H., Bauer, T., Baumjohann, W., Fillius, W., Förster, M., Frey, S., Georgescu, E., Kerr, S. S., Kletzing, C. A., Matsui, H., Puhl-Quinn, P., and Whipple, E. C.: The Electron Drift Instrument on Cluster: overview of first results, Ann. Geophys., 19, 1273-1288, 2001, http://www.ann-geophys.net/19/1273/2001/.

Quinn, J. M., Paschmann, G., Torbert, R. B., Vaith, H., McIlwain, C. E., Haerendel, G., Bauer, O., Bauer, T. M., Baumjohann, W., Fillius, W., Foerster, M., Frey, S., Georgescu, E., Kerr, S. S., Kletzing, C. A., Matsui, H., Puhl-Quinn, P., and Whipple, E. C.: Cluster EDI convection measurements across the high-latitude plasma sheet boundary at midnight, Ann. Geophys., 19, 16691681, 2001,

http://www.ann-geophys.net/19/1669/2001/.

Ridley, A. J., Richmond, A. D., Gombosi, T. I., De Zeeuw, D. L., and Clauer, C. R.: Ionospheric control of the magnetospheric configuration: Thermospheric neutral winds, J. Geophys. Res., 108, 1328, doi:10.1029/2002JA009464, 2003.

Rosenbauer, H., Gruenwaldt, H., Montgomery, M. D., Paschmann,
G., and Sckopke, N.: Heos 2 plasma observations in the distant polar magnetosphere - The plasma mantle, J. Geophys. Res., 80, 2723-2737, 1975.

Ruohoniemi, J. M. and Baker, K. B.: Large-scale imaging of highlatitude convection with Super Dual Auroral Radar Network HF radar observations, J. Geophys. Res., 103, 20 797-20 812, 1998.

Ruohoniemi, J. M. and Greenwald, R. A.: Dependencies of high-latitude plasma convection: Consideration of interplanetary magnetic field, seasonal, and universal time factors in statistical patterns, J. Geophys. Res., 110, A09 204, doi:10.1029/ 2004JA010815, 2005.

Russell, C. T. and Brody, K. I.: Some Remarks on the Position and Shape of the Neutral Sheet, J. Geophys. Res., 72, 6104-6106, 1967.

Sibeck, D. G., Siscoe, G. L., Slavin, J. A., Smith, E. J., Tsurutani, B. T., and Lepping, R. P.: The distant magnetotail's response to a strong interplanetary magnetic field $B_{y}$ - Twisting, flattening, and field line bending, J. Geophys. Res., 90, 4011-4019, 1985.

Sibeck, D. G., Paschmann, G., Treumann, R. A., Fuselier, S. A., Lennartsson, W., Lockwood, M., Lundin, R., Ogilvie, K. W., Onsager, T. G., Phan, T.-D., Roth, M., Scholer, M., Sckopke, N., Stasiewicz, K., and Yamauchi, M.: Chapter 5-Plasma Transfer Processes at the Magnetopause, Space Sci. Rev., 88, 207-283, doi:10.1023/A:1005255801425, 1999.

Siscoe, G. L. and Sanchez, E.: An MHD model for the complete open magnetotail boundary, J. Geophys. Res., 92, 7405-7412, 1987.

Smith, C. W., L'Heureux, J., Ness, N. F., Acuña, M. H., Burlaga, L. F., and Scheifele, J.: The ACE Magnetic Fields Experiment, Space Sci. Rev., 86, 613-632, 1998.

Svenes, K., Lybekk, B., Pedersen, A., and Haaland, S.: Cluster observations of magnetospheric lobe plasma densities for different solar wind conditions - a statistical study, Ann. Geophys., 26, in press, 2008.

Tanaka, T.: Interplanetary magnetic field $B_{y}$ and auroral conductance effects on high-latitude ionospheric convection patterns, J. Geophys. Res., 106, 24 505-24 516, 2001.

Tsyganenko, N. A.: A model of the near magnetosphere with a dawn-dusk asymmetry 1. Mathematical structure, J. Geophys. Res., 107, 1179, doi:10.1029/2001JA000219, 2002a.

Tsyganenko, N. A.: A model of the near magnetosphere with a dawn-dusk asymmetry 2. Parameterization and fitting to observations, J. Geophys. Res., 107, 1176, doi:10.1029/2001JA000220, $2002 b$.

Weimer, D. R.: Improved ionospheric electrodynamic models and application to calculating Joule heating rates, J. Geophys. Res., 110, 5306, doi:10.1029/2004JA010884, 2005.

Weimer, D. R. and King, J. H.: Improved calculations of interplanetary magnetic field phase front angles and propagation time delays, J. Geophys. Res., 113, A01 105, doi:10.1029/ 2007JA012452, 2008.

Weimer, D. R., Ober, D. M., Maynard, N. C., Collier, M. R., McComas, D. J., Ness, N. F., Smith, C. W., and Watermann, J.: Predicting interplanetary magnetic field (IMF) propagation delay times using the minimum variance technique, J. Geophys. Res., 108, 1026, doi:10.1029/2002JA009405, 2003.

Wolf, R. A.: Effects of ionospheric conductivity on convective flow of plasma in the magnetosphere., J. Geophys. Res., 75, 46774698, 1970. 